\title{
Cognitive-Relay-Based Intercell Interference Cancellation in Cellular Systems
}

\author{
Hongbing Cheng, Member, IEEE, and Yu-Dong Yao, Senior Member, IEEE
}

\begin{abstract}
This paper proposes to use a cognitive-relay technique to mitigate intercell interference (ICI) in cellular systems. In the proposed system, several relay stations (RSs) that are equipped with cognitive radio are deployed near the cell boundary. The base station (BS) and the RS in each cell operate in the same spectrum band as primary and secondary transmitters, respectively. Once an interference-limited mobile station (MS) requests an RS for assistance, the RS senses the spectrum band and accesses a spectrum hole to forward its received signal (and interference) to the MS. At the receiver of the MS, optimum combining is employed to combine the original signal that is received from the BS and the relayed signal to cancel the ICI. The system performance is analyzed in terms of the outage capacity and the average capacity considering the impact of the availability of cognitive-relay channels and the link quality between the RS and the MS. The location and the coverage radius of the RS are designed based on the requirement of the RS-MS link quality. Finally, simulation results are given to validate the theoretical analysis and to show the capacity improvement due to the ICI cancellation with the assistance of cognitive relay.
\end{abstract}

Index Terms-Cognitive relay, intercell interference (ICI), optimum combining.

\section{INTRODUCTION}

$\mathbf{I}$ N CELLULAR communication systems, one of the major factors degrading system performance is intercell interference (ICI), which is caused by neighboring cells using the same frequency band; this is known as cochannel interference (CCI). The ICI experienced by mobile stations (MSs), particularly the MS near the cell boundary, significantly impacts the link quality (often measured in terms of the bit error rate or the outage probability) and limits the system capacity. Many techniques have been proposed to mitigate the ICI. However, the conventional methods usually compromise spectral efficiency to reduce the ICI, such as reducing the frequency reuse factor, spreading spectrum, and frequency hopping [1]-[3]. To mitigate the ICI without reducing the spectral efficiency of cellular systems, single-input-multiple-output (SIMO) or multiple-input-multiple-output (MIMO) techniques with optimum combing have been proposed [4], [5]. The performance improvement obtained in SIMO and MIMO systems through optimum combining has been analyzed and simulated by many

Manuscript received May 22, 2009; revised August 31, 2009. First published January 22, 2010; current version published May 14, 2010. The review of this paper was coordinated by Dr. H. Harada.

The authors are with the Wireless Information System Engineering Laboratory, Department of Electrical and Computer Engineering, Stevens Institute of Technology, Hoboken, NJ 07030 USA (e-mail: hcheng@stevens.edu; yyao@stevens.edu).

Color versions of one or more of the figures in this paper are available online at http://ieeexplore.ieee.org.

Digital Object Identifier 10.1109/TVT.2010.2040399 researchers [6], [7]. However, the space and resource limits are a major obstacle in practice for the deployment of multiple antennas at an MS.

Multihop relay is a promising technique for cellular systems to enhance throughput and extend coverage [8]. Its basic idea is to employ a relay station (RS) between a base station (BS) and an MS to improve the performance of signal transmissions. Two types of relay schemes have been proposed for cellular systems. One is the conventional relaying scheme without cooperative diversity, where the MS can only receive the signal that is forwarded by the RS. This type of scheme is used only for pathloss compensations or to divert traffic from possibly congested areas to lower traffic areas [9], [10]. The other is the cooperative relay scheme, where the MS can receive both the signals that are originally transmitted by the BS and forwarded by the RS [11], [12]. When the BS and the RS transmit in orthogonal channels (different time slots or frequency bands), the MS can combine the two signals to obtain the diversity gain. Much research has proposed to use decode-and-forward (DF) relay to combat the ICI [13], [14]. The use of a DF relay improves system performance due to the diversity gain and resource allocation. However, the ICI cannot be canceled since no ICI signal is relayed from the DF RS, which limits the potential of performance improvement. It is interesting to notice that if the amplify-and-forward (AF) relay is used and both the desired signal and the ICI signal are relayed, the combination of the received signals from the $\mathrm{BS}$ and the RS can be used to cancel the ICI, which is similar to the SIMO system with two receive antennas. This introduces more opportunities to improve the spectral efficiency of cellular systems using relay techniques. However, the use of a relay generally requires additional time or frequency resources for the communications between the RS and the MS.

As has been reported, most radio systems do not utilize all the assigned frequency bands all the time [15]. The unused frequency bands are called spectrum holes. A cognitive radio technique has recently been proposed to improve spectrum utilization by allowing secondary users to access the spectrum holes [16]. Recent research efforts have studied the application of cooperative relay techniques in cognitive radio systems, such as cooperative spectrum sensing and spectrum sharing [17]. The concept of cognitive relay has been proposed by several researchers for different scenarios [18]-[20]. The primary idea is to use cognitive radio nodes as a relay to assist the communications of primary nodes. Since the relay nodes cognitively utilize the spectrum assigned to but unused by the primary nodes, it does not incur additional resource consumption. The use of cognitive relay in cellular systems was considered in [21], 
and the paper only considered a noise-limited environment without ICI. It assumes that the RS utilizes the spectrum hole in an ultrahigh-frequency band or industry, industrial, scientific, and medical (ISM) bands, and no interference exists between the RS-MS link and the BS-MS link.

In this paper, we propose a cognitive-relay-based cellular system by applying the cognitive-relay technique to mitigate the ICI. In the proposed scheme, several RSs equipped with cognitive radio are deployed around each cell's boundary. Here, cognitive relay means that the RS can detect and recognize the vacant channels assigned to but unused by the primary communications between the BS and the MS by the use of existing spectrum-sensing methods and cognitively access such channels to transmit signals [22], [23]. The RS and the MS both receive the signal from the home cell BS and the ICI from other cochannel BS in the downlink channel. Instead of using other bands as in [21], we assume that the cognitive RS in each cell operates in the spectrum band that is assigned for the primary downlink communications (i.e., BS-MS links) in the cell. If an interference-limited MS requires a relay from the RS, the RS will sense the spectrum band and find an unutilized channel to amplify and forward its received signal and interference to the MS. The MS combines the received signals and interference from the BS and the RS to perform the ICI mitigation. We study the outage capacity of the cognitive-relay-based cellular system and identify two parameters that impact system performance. One is the availability probability of the cognitive-relay channel, and the other is the link quality between the RS and the MS (determined by path loss and ICI). Theoretical models are formulated to quantify and derive the two parameters. The locations and the coverage of the RS are designed based on the requirement of the link quality between the RS and the MS. Theoretical and simulation results are provided to show the effectiveness of the proposed cognitive-relay-based ICI-mitigation scheme with various system parameters.

To summarize, the contributions of this paper are threefold. First, we propose and design the cognitive-relay-based cellular system to mitigate the ICI. Second, the performance of the proposed system is analyzed considering the impact of the availability of the cognitive-relay channel and the RS-MS link quality. Third, the coverage radius of the RS at different locations is derived, which provides a guideline for deploying the $\mathrm{RS}$ in a cell. The remainder of this paper is organized as follows. In Section II, we describe the proposed cellular system model with cognitive relay and optimum combining. The performance of the proposed system is analyzed in Section III, and the simulation results are given in Section IV. Section V summarizes our conclusions.

\section{SYSTEM MOdEL}

We consider a downlink fading channel in a multicell environment, where each cell is interfered with by $N_{I}$ cochannel cells. Different users in one cell are allocated with orthogonal channels to avoid intracell interference, such as using timedivision multiple access, code-division multiple access, or orthogonal frequency-division multiple access. However, the use of the same channel in different cells may cause ICI. The home

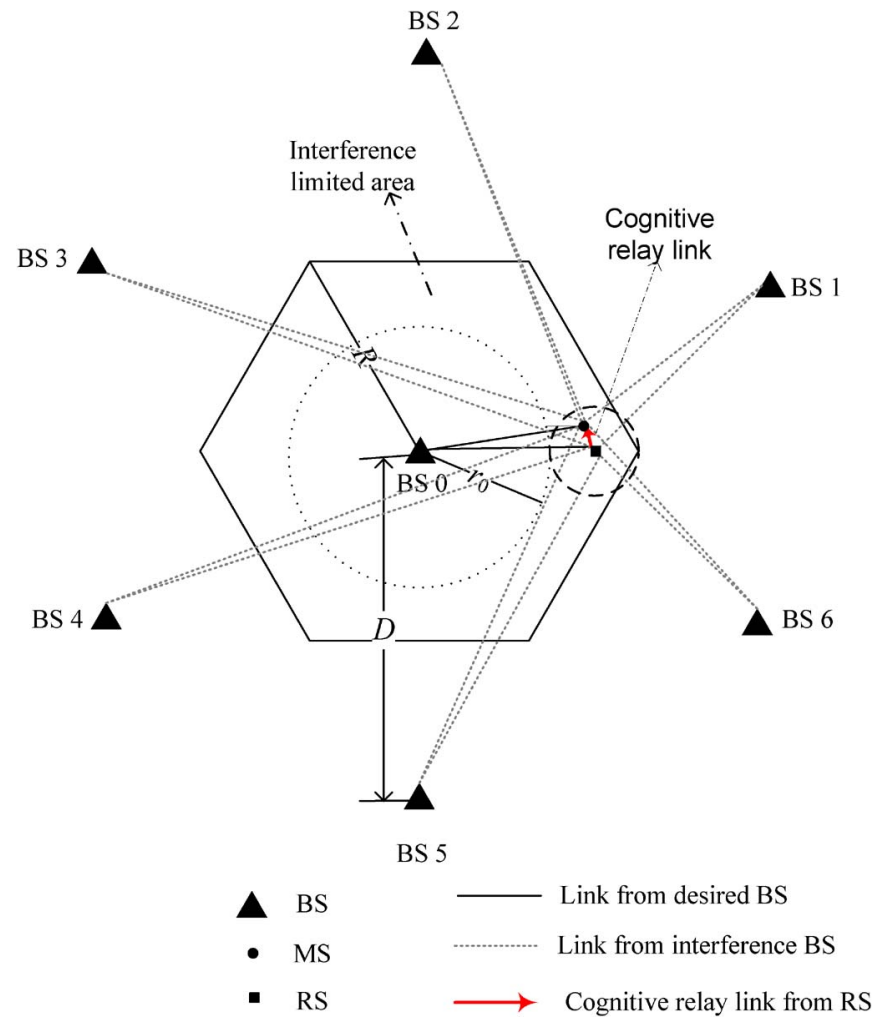

Fig. 1. Cellular system model with cognitive relay.

cell is denoted as cell 0 , and the interference cells are from cell 1 to $N_{I}$. Correspondingly, the BSs are denoted as BS 0 to $N_{I}$.

To help the MS against the ICI, several RSs are deployed around the cell boundary of each cell. The RS and the MS are equipped with two types of radio functionalities. One is the primary radio to communicate with the BS. The other is the secondary radio, or the so-called the cognitive radio, for opportunistic communications between the RS and the MS. For the downlink transmission, the RS and the MS receive signals from all BSs through the primary radio. If an MS suffers from strong ICI and has a low-quality link with the BS, it will need assistance from the RS. One of the RSs is selected to be the MS's associated RS, and it will relay its received signal to the MS through cognitive radio. Specifically, it will first sense the spectrum band that is allocated to the primary radio to detect a spectrum hole (a vacant channel). Many existing spectrumsensing techniques, such as energy detection and likelihood detection, can be applied for the detection [22], [23]. Once a spectrum hole is detected, the RS will cognitively adjust its radio parameters to communicate with the MS through the spectrum hole. This is called cognitive relay. Fig. 1 illustrates our proposed cellular network with cognitive relay.

An MS may operate in noise-limited regime or interferencelimited regime. Generally, the operation condition is quantified in terms of the interference-to-noise ratio $\psi=P_{\mathrm{ICI}} / \sigma_{0}^{2}$, where $P_{\mathrm{ICI}}$ is the power of the ICI, and $\sigma_{0}^{2}$ is the power of the background noise [24]. If $\psi>1$, the ICI dominates over noise. When $\psi \gg 1$, the system is regarded as interference limited. In this paper, we assume that all the BSs have the same transmit power. The ICI power of an MS is totally determined by its location and increases with the increase in its distance to the 
home cell BS. Therefore, we simply classify the MS with $r \leq r_{0}$ as a noise-limited MS and the MS with $r>r_{0}$ as an interference-limited MS, respectively, where $r$ is the distance between the MS and its home BS, and $r_{0}$ is a given threshold.

We assume that only the interference-limited MS will request the cognitive relay, and all the RSs are interference-limited since they are deployed near the cell boundary. The RS should be appropriately deployed so that each interference-limited MS is covered by at least one RS. The location and the coverage of the RS will be discussed in Section III.

To simplify the model, the background noises at the interference-limited MS and RS are assumed to be negligible. The received signals of an interference-limited MS and its associated RS through the primary radio are written as

$$
\begin{gathered}
y_{\mathrm{b}, \mathrm{m}}=\sqrt{P_{\mathrm{tb}}} g_{\mathrm{b}_{0}, \mathrm{~m}} h_{\mathrm{b}_{0}, \mathrm{~m}} x_{0}+\sum_{i=1}^{N_{I}} \sqrt{P_{\mathrm{tb}}} g_{\mathrm{b}_{i}, \mathrm{~m}} h_{\mathrm{b}_{i}, \mathrm{~m}} x_{i} \\
y_{\mathrm{b}, \mathrm{r}}=\sqrt{P_{\mathrm{tb}}} g_{\mathrm{b}_{0}, \mathrm{r}} h_{\mathrm{b}_{0}, \mathrm{r}} x_{0}+\sum_{i=1}^{N_{I}} \sqrt{P_{\mathrm{tb}}} g_{\mathrm{b}_{i}, \mathrm{r}} h_{\mathrm{b}_{i}, \mathrm{r}} x_{i}
\end{gathered}
$$

respectively, where $P_{\mathrm{tb}}$ is the transmit power of each BS. $x_{0}$ is the desired signal transmitted from the BS of the home cell to the MS, and $x_{i}$, with $i=1,2, \ldots, N_{I}$, is the ICI signal from the BS of cell $i$, i.e., the $i$ th BS. $h_{\mathrm{b}_{i}, \mathrm{~m}}$ and $h_{\mathrm{b}_{i}, \mathrm{r}}$ are complex Gaussian-distributed random variables representing the flat-fading channels of the $i$ th BS-MS link and BS-RS link, respectively, which are assumed to have unit power, i.e., $E\left[\left|h_{\mathrm{b}_{i}, \mathrm{~m}}\right|^{2}\right]=1$ and $E\left[\left|h_{\mathrm{b}_{i}, \mathrm{r}}\right|^{2}\right]=1 . g_{\mathrm{b}_{i}, \mathrm{~m}}$ and $g_{\mathrm{b}_{i}, \mathrm{r}}$ are the propagation path losses of the $i$ th BS-MS link and BS-RS link, respectively, which are calculated as

$$
\begin{aligned}
g_{\mathrm{b}_{i}, \mathrm{~m}} & =\sqrt{\left|g_{0}\right|^{2} d_{\mathrm{b}_{i}, \mathrm{~m}}^{-n}} \\
g_{\mathrm{b}_{i}, \mathrm{r}} & =\sqrt{\left|g_{0}\right|^{2} d_{\mathrm{b}_{i}, \mathrm{r}}^{-n}}
\end{aligned}
$$

for $i=0,1, \ldots, N_{I}$, where $\left|g_{0}\right|^{2}$ is the reference channel gain at the distance of $1 \mathrm{~m} . d_{\mathrm{b}_{i}, \mathrm{~m}}$ and $d_{\mathrm{b}_{i}, \mathrm{r}}$ are the communication distances of the $i$ th BS-MS link and BS-RS link, respectively. Notice that $d_{\mathrm{b}_{0}, \mathrm{~m}}=r$ and that $n$ is the path-loss factor.

After receiving the signal with ICI, the MS sends a relay request to its associated RS. The RS then detects the spectrum band to sense if there is a vacant channel. If no channel is found, the request is discarded. Otherwise, the RS amplifies and relays its received signal, including the desired signal and ICI, to the MS through the vacant channel following the AF relay protocol. The received signal at the MS through this cognitiverelay channel is

$$
y_{\mathrm{r}, \mathrm{m}}=\sqrt{\alpha P_{\mathrm{tb}}} g_{\mathrm{r}, \mathrm{m}} h_{\mathrm{r}, \mathrm{m}} g_{\mathrm{b}_{0}, \mathrm{r}} h_{\mathrm{b}_{0}, \mathrm{r}} x_{0}+I_{\mathrm{r}, \mathrm{m} 1}+I_{\mathrm{r}, \mathrm{m} 2}
$$

with

$$
\begin{aligned}
& I_{\mathrm{r}, \mathrm{m} 1}=\sqrt{\alpha P_{\mathrm{tb}}} g_{\mathrm{r}, \mathrm{m}} h_{\mathrm{r}, \mathrm{m}} \sum_{i=1}^{N_{I}} g_{\mathrm{b}_{i}, \mathrm{r}} h_{\mathrm{b}_{i}, \mathrm{r}} x_{i} \\
& I_{\mathrm{r}, \mathrm{m} 2}=\sqrt{P_{\mathrm{tb}}} \sum_{i=1}^{N_{I}} g_{\mathrm{b}_{i}, \mathrm{~m}} \tilde{h}_{\mathrm{b}_{i}, \mathrm{~m}} \tilde{x}_{i}
\end{aligned}
$$

where $\alpha$ is the power amplify factor of the RS, which is assumed to be fixed over small-scale channel fading (i.e., fixedgain relay) and calculated as [25], [26]

$$
\alpha=\frac{P_{\mathrm{tr}}}{P_{\mathrm{tb}} \sum_{i=0}^{N_{I}} g_{\mathrm{b}_{i}, \mathrm{r}}^{2}}
$$

where $P_{\mathrm{tr}}$ is the transmit power of the RS. $g_{\mathrm{r}, \mathrm{m}}$ and $h_{\mathrm{r}, \mathrm{m}}$ are the propagation path loss and the small-scale channel fading between the RS and the MS. Similarly

$$
g_{\mathrm{r}, \mathrm{m}}=\sqrt{\left|g_{0}\right|^{2} d_{\mathrm{r}, \mathrm{m}}^{-n}}
$$

where $d_{r, m}$ is the distance between the MS and its associated RS. The received signal in (5) contains two parts of ICI, i.e., $I_{\mathrm{r}, \mathrm{m} 1}$ and $I_{\mathrm{r}, \mathrm{m} 2} . I_{\mathrm{r}, \mathrm{m} 1}$ is the ICI that is intentionally transmitted from the RS to the MS, which will be used to cancel the ICI at the MS, and $I_{\mathrm{r}, \mathrm{m} 2}$ is the inevitable ICI that is transmitted by the other BS over the relay channel. $\tilde{h}_{\mathrm{b}_{i}, \mathrm{~m}}$ is the smallscale fading of the relay channel between the $i$ th BS and the MS. $\tilde{x}_{i}$ is the signal transmitted by the $i$ th BS over the relay channel. Notice that $\tilde{h}_{\mathrm{b}_{i}, \mathrm{~m}}$ and $\tilde{x}_{i}$ are independent of $h_{\mathrm{b}_{i}, \mathrm{~m}}$ and $x_{i}$, respectively, since the cognitive-relay channel is orthogonal to primary channels.

The ICI $I_{\mathrm{r}, \mathrm{m} 2}$ exists in the cognitive-relay channel because the RS can only detect if a channel is occupied by primary radios in its own cell but cannot detect if it is occupied in neighboring cochannel cells. Correspondingly, the transmission of the RS will also cause ICI to the MS in neighboring cochannel cells. To protect the primary communications in neighboring cells, we have to constrain the transmit power of the RS so that its interference to neighboring cells is tolerable. This constraint is quantified in terms of the ratio between the maximum ICI power caused by the RS and the minimum signal power in neighboring cells and is written as

$$
\frac{P_{\mathrm{tr}} d_{\min }^{-n}}{P_{\mathrm{tb}} R^{-n}} \leq \lambda
$$

where $d_{\min }$ is the minimum distance from the RS to the MS in neighboring cells, and $R$ is the cell radius. $\lambda$ is the allowed interference-to-signal ratio (ISR) at the MS. Assuming that each RS always transmits with the maximum power, we have

$$
\alpha=\lambda \frac{R^{-n}}{d_{\min }^{-n} \sum_{i=0}^{N_{I}} g_{\mathrm{b}_{i}, \mathrm{r}}^{2}} .
$$

The received signals of the MS from the BS and the RS, i.e., (1) and (5), can be represented in a vector form as

$$
\mathbf{y}=\sqrt{P_{\mathrm{tb}}}\left[\mathbf{c}_{0} x_{0}+\sum_{i=1}^{N_{I}} \mathbf{c}_{i} x_{i}\right]+\mathbf{I}
$$

with $\mathbf{y}=\left[y_{\mathrm{b}, \mathrm{m}}, y_{\mathrm{r}, \mathrm{m}}\right]^{T}, \mathbf{c}_{i}=\left[g_{\mathrm{b}_{i}, \mathrm{~m}} h_{\mathrm{b}_{i}, \mathrm{~m}}, \sqrt{\alpha} g_{\mathrm{b}_{i}, \mathrm{r}} h_{\mathrm{b}_{i}, \mathrm{r}} g_{\mathrm{r}, \mathrm{m}} h_{\mathrm{r}, \mathrm{m}}\right]^{T}$ for $i=0,1, \ldots, N_{I}$, and $\mathbf{I}=\left[0, I_{\mathrm{r}, \mathrm{m} 2}^{T}\right]$.

At the receiver, the two received signals will be combined to cancel the ICI and improve the system performance. The 
combination output is

$$
z=\mathbf{w}^{\mathbf{H}} \mathbf{y}
$$

where $\mathbf{w}$ is the combining vector. To maximize the SIR, the optimum combining vector is given by [6]

$$
\mathbf{w}_{\mathrm{opt}}=\mathbf{\Omega}^{-1} \mathbf{c}_{0}
$$

where

$$
\begin{aligned}
\boldsymbol{\Omega} & =\sum_{i=1}^{n_{i}} \mathbf{c}_{i} \mathbf{c}_{i}^{h}+\frac{e\left\{\mathbf{I I}^{h}\right\}}{p_{\mathrm{tb}}} \\
& =\sum_{i=1}^{n_{i}}\left(\mathbf{c}_{i} \mathbf{c}_{i}^{h}+\left[\begin{array}{cc}
0 & 0 \\
0 & g_{\mathrm{b}_{i}, \mathrm{~m}}^{2}
\end{array}\right]\right) .
\end{aligned}
$$

\section{PERformance ANALysis}

\section{A. Outage Capacity Analysis}

Outage capacity is an important measure of a communication system, particularly in a slow-fading channel environment, where the delay requirement is small compared with the coherent time. The definition of outage capacity is given as follows [27]:

$$
C_{\epsilon}=\arg \max _{C_{\mathrm{th}}}\left\{P_{\mathrm{out}}\left(C_{\mathrm{th}}\right) \leq \epsilon\right\}
$$

where $C_{\epsilon}$ is the outage capacity, and $\epsilon$ is the target outage probability. $P_{\text {out }}\left(C_{\mathrm{th}}\right)$ is the outage probability with a threshold capacity $C_{\mathrm{th}}$, which is calculated as

$$
P_{\text {out }}\left(C_{\text {th }}\right)=\operatorname{Pr}\left\{C(\gamma) \leq C_{\text {th }}\right\}
$$

where $C(\gamma)$ is the instantaneous capacity with a given SIR $\gamma$ calculated as

$$
C(\gamma)=\log _{2}(1+\gamma)
$$

Considering an interference-limited MS in our cellular system model, if the MS fails to request the relay, the instantaneous SIR is

$$
\gamma_{\text {norelay }}=\frac{\left|g_{\mathrm{b}_{0}, \mathrm{~m}} h_{\mathrm{b}_{0}, \mathrm{~m}}\right|^{2}}{\sum_{i=1}^{N_{I}}\left|g_{\mathrm{b}_{i}, \mathrm{~m}} h_{\mathrm{b}_{i}, \mathrm{~m}}\right|^{2}} .
$$

If the MS obtains the help of relay and conducts the optimum combining at the receiver, the instantaneous output SIR is

$$
\gamma_{\mathrm{oc}}=\mathbf{c}_{0}^{H} \boldsymbol{\Omega}^{-1} \mathbf{c}_{0}=\hat{\mathbf{c}}_{0}^{H} \hat{\boldsymbol{\Omega}}^{-1} \hat{\mathbf{c}}_{0}
$$

where $\quad \hat{\mathbf{c}}_{i}=\left[g_{\mathrm{b}_{i}, \mathrm{~m}} h_{\mathrm{b}_{i}, \mathrm{~m}}, g_{\mathrm{b}_{i}, \mathrm{r}} h_{\mathrm{b}_{i}, \mathrm{r}}\right]^{T} \quad$ for $\quad i=0,1,2, \ldots$, $N_{I}$, and

$$
\hat{\boldsymbol{\Omega}}=\hat{\mathbf{C}}_{I} \hat{\mathbf{C}}_{I}^{H}+\left[\begin{array}{cc}
0 & 0 \\
0 & \delta \sum_{i=1}^{N} g_{\mathrm{b}_{i}, \mathrm{~m}}^{2}
\end{array}\right]
$$

with $\hat{\mathbf{C}}_{I}=\left[\hat{\mathbf{c}}_{1}, \hat{\mathbf{c}}_{2}, \ldots, \hat{\mathbf{c}}_{N_{I}}\right]$. The parameter $\delta$ is defined as

$$
\delta=\frac{1}{\alpha\left|g_{\mathrm{r}, \mathrm{m}} h_{\mathrm{r}, \mathrm{m}}\right|^{2}}
$$

which is the reciprocal of the RS-MS link gain (the power amplify factor at the transmitter of the link multiplied by the channel gain). We, thus, use $\delta$ to quantify the RS-MS link quality.

The outage probability of the MS with cognitive relay is

$$
P_{\text {out }}\left(C_{\mathrm{th}}\right)=(1-p) P_{\text {out } 1}\left(C_{\mathrm{th}}\right)+p P_{\text {out } 2}\left(C_{\mathrm{th}}\right)
$$

where $P_{\text {out } 1}\left(C_{\text {th }}\right)=\operatorname{Pr}\left(\gamma_{\text {norelay }} \leq \gamma_{\text {th }}\right)$, and $P_{\text {out } 2}\left(C_{\text {th }}\right)=$ $\operatorname{Pr}\left(\gamma_{\mathrm{oc}} \leq \gamma_{\mathrm{th}}\right) \cdot \gamma_{\mathrm{th}}$ is the threshold SIR, and $\gamma_{\mathrm{th}}=2^{C_{\mathrm{th}}}-1$. $p$ is the availability probability of the cognitive-relay channel, which will be discussed in Section III-C.

Assuming that the small-scale fading of all the channels follows a Rayleigh distribution and $g_{\mathrm{b}_{1}, \mathrm{~m}} \geq g_{\mathrm{b}_{2}, \mathrm{~m}} \geq \cdots \geq$ $g_{\mathrm{b}_{N_{I}}, \mathrm{~m}}$, the outage probability without relay is [6]

$$
P_{\text {out } 1}\left(C_{\mathrm{th}}\right)=\frac{\operatorname{det}\left[\mathcal{C}_{1}\left(\gamma_{\mathrm{th}}\right)\right]}{\operatorname{det}\left(\mathcal{G}_{1}\right)}
$$

where $\mathcal{G}$ is an $N_{I} \times N_{I}$ matrix given by

$$
\left\{\mathcal{G}_{1}\right\}_{i, j}=g_{\mathrm{b}_{i}, \mathrm{~m}}^{2(j-1)} \text {. }
$$

$\mathcal{C}_{1}(x)$ is an $N_{I} \times N_{I}$ matrix given by

$$
\left\{\mathcal{C}_{1}(x)\right\}_{i, j}= \begin{cases}g_{\mathrm{b}_{j}, \mathrm{~m}}^{2(i-1)}, & i=1, \ldots, N_{I}-1 \\ \frac{x g_{\mathrm{b}_{j}, \mathrm{~m}}^{2 N_{I}}}{1+x g_{\mathrm{b}_{j}, \mathrm{~m}}^{2}}, & i=N_{I} j=1, \ldots, N_{I} .\end{cases}
$$

When relay is available, it is hard to derive a closed-form outage probability. Some analyses are given as follows. Since the distance between the BS and the MS is much larger than the distance between the RS and the MS, we approximate that $d_{\mathrm{b}_{i}, r} \approx$ $d_{\mathrm{b}_{i}, m}$, and therefore, $g_{\mathrm{b}_{i}, \mathrm{r}} \approx g_{\mathrm{b}_{i}, \mathrm{~m}}$ for $i=0,1,2, \ldots, N_{I}$. The SIR with relay is, thus, approximated by

$$
\gamma_{\mathrm{oc}} \approx g_{\mathrm{b}_{0}, \mathrm{~m}}^{2} \mathbf{h}_{0}^{H} \tilde{\boldsymbol{\Omega}}^{-1} \mathbf{h}_{0}
$$

with $\mathbf{h}_{0}=\left[h_{\mathrm{b}_{0}, \mathrm{~m}}, h_{\mathrm{b}_{0}, \mathrm{r}}\right]^{T}$ and $\tilde{\boldsymbol{\Omega}}=\mathbf{H}_{\mathbf{I}} \mathbf{G}_{\mathbf{I}} \mathbf{H}_{\mathbf{I}}^{H}$, where $\mathbf{H}_{I}=$ $\left[\mathbf{h}_{1}, \mathbf{h}_{2}, \ldots, \mathbf{h}_{N_{I}}\right], \mathbf{h}_{i}=\left[h_{\mathrm{b}_{i}, \mathrm{~m}}, h_{\mathrm{b}_{i}, \mathrm{r}}+\sqrt{\delta}\right]^{T}$ for $i=1,2, \ldots$, $N_{I}$, and

$$
\mathbf{G}_{\mathbf{I}}=\left[\begin{array}{cccc}
g_{\mathrm{b}_{1}, \mathrm{~m}}^{2} & 0 & \cdots & 0 \\
0 & g_{\mathrm{b}_{2}, \mathrm{~m}}^{2} & \cdots & 0 \\
\vdots & \vdots & \ddots & \vdots \\
0 & 0 & \cdots & g_{\mathrm{b}_{N_{I}}, \mathrm{~m}}^{2}
\end{array}\right]
$$

When $\delta=0$, the SIR in (27) becomes the same as the SIR of a $1 \times 2$ SIMO system with optimum combining. Following [6], assuming that $N_{I} \geq 2$ and $g_{\mathrm{b}_{1}, \mathrm{~m}} \geq g_{\mathrm{b}_{2}, \mathrm{~m}} \geq \cdots \geq g_{\mathrm{b}_{N_{I}}, \mathrm{~m}}$, the outage probability is calculated as

$$
\left.P_{\text {out } 2}\left(C_{\text {th }}\right)\right|_{\delta=0}=-\frac{\operatorname{det}\left[\mathcal{C}_{2}\left(\gamma_{\text {th }}\right)\right]}{\operatorname{det}\left(\mathcal{G}_{2}\right)}
$$

where $\mathcal{G}_{2}$ is an $N_{I} \times N_{I}$ matrix given by

$$
\left\{\mathcal{G}_{2}\right\}_{i, j}=g_{\mathrm{b}_{i}, \mathrm{~m}}^{2(j-1)} \text {. }
$$


$\mathcal{C}_{2}(x)$ is an $N_{I} \times N_{I}$ matrix given by

$$
\mathcal{C}_{2}(x)= \begin{cases}g_{\mathrm{b}_{j}, \mathrm{~m}}^{2(i-1)}, & i=1, \ldots, N_{I}-2 \\ g_{\mathrm{b}_{j}, \mathrm{~m}}^{2\left(N_{I}-1\right)}, & j=1, \ldots, N_{I} \\ \frac{x g_{\mathrm{b}_{j}, \mathrm{~m}}^{2\left(N_{I}-1\right)}}{1+x g_{\mathrm{b}_{j}, \mathrm{~m}}^{2}}, & i=N_{I}-1 j=1, \ldots, N_{I}\end{cases}
$$

Notice that if there exist any two equal $g_{\mathrm{b}_{i}, \mathrm{~m}}$ for different $i$, (24) and (29) become 0/0 types of limits. The L'Hôpital's rule should be used to get the outage probability as in [28].

Equation (29) is the best performance bound of the relay system and can be obtained only when the RS-MS channel is perfect, i.e., $\delta=0$. As defined in (22), $\delta$ is an indicator of the RS-MS link quality. When $\delta$ is increased, i.e., the RS-MS link gets worse, the SIR is degraded, and the outage probability is increased. To make sure that the cognitive relay efficiently helps mitigating the ICI, we should carefully select the position of the associated RS for each MS so that $\delta$ is so small that we can obtain the best performance, i.e.,

$$
\left.P_{\text {out } 2}\left(C_{\mathrm{th}}\right) \approx P_{\text {out } 2}\left(C_{\mathrm{th}}\right)\right|_{\delta=0} .
$$

\section{B. Average Capacity Analysis}

Average capacity is another performance measure of communication systems, particularly in fast-fading channel environments, which is defined as

$$
\bar{C}=\int_{\gamma} C(\gamma) f(\gamma) d \gamma
$$

where $f(\gamma)$ is the probability density function of SIR $\gamma$.

In the cognitive-relay cellular systems, the cognitive relay is available for ICI cancellation with a probability $p$. Therefore, the average capacity is calculated as

$$
\bar{C}=(1-p) \bar{C}_{1}+p \bar{C}_{2}
$$

where

$$
\begin{aligned}
\bar{C}_{1} & =\int_{0}^{+\infty} \log _{2}\left(1+\gamma_{\text {norelay }}\right) f\left(\gamma_{\text {norelay }}\right) d \gamma_{\text {norelay }} \\
\bar{C}_{2} & =\int_{0}^{+\infty} \log _{2}\left(1+\gamma_{\mathrm{oc}}\right) f\left(\gamma_{\mathrm{oc}}\right) d \gamma_{\mathrm{oc}} .
\end{aligned}
$$

\section{Availability Probability of the Cognitive-Relay Channel}

Here, we establish a model to derive the availability probability of the relay channel, i.e., $p$, which is an important factor impacting the outage capacity, as shown in (23), and the average capacity, as shown in (34). Assume that there are $N_{c}$ orthogonal channels that are allocated to each cell and that the user number in each cell is $N_{u}$, with $N_{u} \leq N_{c}$. Each user is randomly allocated with one channel for communications with the BS.
Different users' channels are orthogonal. The loading factor is defined as the ratio between the user number and the available channel number, i.e.,

$$
\rho=\frac{N_{u}}{N_{c}}
$$

It is worth noting that each user does not occupy the allocated channel all the time. In other words, there is an activity factor $\beta$ for each user, which is the probability that the user is active. Therefore, the probability that there are $K$ vacant channels that are unoccupied by the primary communications is

$$
p_{\mathrm{ch}}(K)= \begin{cases}\tilde{p}_{\mathrm{ch}}(K), & (1-\rho) N_{c} \leq K \leq N_{c} \\ 0, & K<(1-\rho) N_{c} \text { or } K>N_{c}\end{cases}
$$

where $\tilde{p}_{\mathrm{ch}}(K)=\mathrm{C}_{\rho N_{c}}^{N_{c}-K} \beta^{N_{c}-K}(1-\beta)^{K-(1-\rho) N_{c}}$, and $\mathrm{C}_{n}^{k}=$ $n ! / k !(n-k) !$

The number of interference-limited users is denoted as $N_{u i}$. If all the MSs are uniformly distributed in the home cell, $N_{u i}$ is calculated as

$$
N_{u i}=\frac{S_{i}}{S} N_{u}=\left(1-\frac{2 \pi}{3 \sqrt{3}} \frac{r_{0}^{2}}{R^{2}}\right) N_{u}
$$

where $S$ is the area of a whole cell, and $S_{i}$ is the area of the interference-limited part of the cell. $r_{0}$ is the interferencelimited threshold distance, as defined in Section II, and $R$ is the cell radius. Notice that $N_{u i}$ must be rounded off to the nearest integer.

The probability that there are $M$ active interference-limited users requesting cognitive-relay channels is

$$
p_{\text {req }}(M)= \begin{cases}\mathrm{C}_{N_{u i}}^{M} \beta^{M}(1-\beta)^{N_{u i}-M}, & M \leq N_{u i} \\ 0, & M>N_{u i} .\end{cases}
$$

The $M$ active interference-limited users will compete for the $K$ available vacant channels. Assuming that the $K$ channels are randomly allocated to the $M$ users, the probability that one relay channel is available for a given user is

$$
p_{\mathrm{a}}(K, M)= \begin{cases}1, & M \leq K \\ \frac{K}{M}, & M>K\end{cases}
$$

By averaging over all $K$ and $M$, the average availability probability of the cognitive-relay channel for a given user is

$$
p=\sum_{K=(1-\rho) N_{c}}^{N_{c}} \sum_{M=0}^{N_{u i}} p_{\text {ch }}(K) p_{\text {req }}(M) p_{\mathrm{a}}(K, M) .
$$

Considering (38)-(42), the availability probability $p$ in a cellular system is a function of the following parameters: $N_{c}$, $\rho, \beta$, and $r_{0}$.

\section{Location and Coverage of the RS}

As mentioned in Section III-A, the effectiveness of cognitive relay depends on the link quality between the RS and the MS. Therefore, we need to carefully determine the location and the coverage of the RS so that the RS-MS link quality is good 
enough. We assume that the RS-MS link can be regarded as perfect if

$$
\operatorname{Pr}\left(\delta>\delta_{0}\right) \leq \varepsilon
$$

where $\delta_{0}$ is a threshold, which indicates the quality requirement of the RS-MS link.

Since $\left|h_{\mathrm{r}, \mathrm{m}}\right|^{2}$ follows an exponential distribution, the above constraint is rewritten as

$$
g_{\mathrm{r}, \mathrm{m}}^{2} \geq \frac{1}{\alpha \hat{\delta}_{0}}
$$

where $\hat{\delta}_{0}=-\delta_{0} \ln (1-\varepsilon)$.

As described in Section II, the maximum transmit power of an RS is constrained by its interference. By inserting (11) in (44), we have

$$
g_{\mathrm{r}, \mathrm{m}}^{2} \geq \frac{d_{\mathrm{min}}^{-n} \sum_{i=0}^{N_{I}} g_{\mathrm{b}_{i}, \mathrm{r}}^{2}}{\lambda R^{-n} \hat{\delta}_{0}} .
$$

Therefore

$$
d_{\mathrm{r}, \mathrm{m}} \leq \hat{\lambda} \frac{d_{\min }}{R}\left(\sum_{i=0}^{N_{I}} d_{\mathrm{b}_{i}, \mathrm{r}}^{-4}\right)^{-1 / 4}
$$

where $\hat{\lambda}=\sqrt[4]{\lambda \hat{\delta}_{0}}$. Notice that the value of the right-hand side in the above inequality is determined only by the location of the RS. Therefore, (46) gives the maximum distance that an RS can reach if the RS's location is given. In other words, for an RS at a given location, its coverage radius is calculated as

$$
R_{c}=\hat{\lambda} \frac{d_{\min }}{R}\left(\sum_{i=0}^{N_{I}} d_{\mathrm{b}_{i}, \mathrm{r}}^{-4}\right)^{-1 / 4} .
$$

\section{Simulation Results}

Simulation results are presented here to validate the theoretical analysis and demonstrate the performance advantage of the cognitive-relay-based cellular systems. In the simulations, we consider the ICI from six nearest interference cells using the same frequency band as the home cell, as shown in Fig. 1. Without loss of generality, we set the cell radius $R=1$. We consider an urban area cellular network and assume that the path-loss factor is $n=4$. The reuse distance $D$, i.e., the distance between BS 0 and the neighboring cochannel $\mathrm{BS}$, is determined by the cluster size $N$ and calculated as [29]

$$
D / R=\sqrt{3 N} .
$$

For convenience, we establish a polar coordinate system with BS 0 as the pole and the line from BS 0 to BS 1 as the polar axis. The location of BS 0 is, thus, represented as $(0,0)$, and the location of BS $i$ is $(D,((i-1) \pi / 3))$ for $i=1,2, \ldots, 6$. The location of an MS is represented as $(r, \theta)$, and the location of an RS is represented as $\left(r^{\prime}, \theta^{\prime}\right)$.

First, we investigate the system performance when the cognitive-relay channel is always available, i.e., $p=1$. Fig. 2

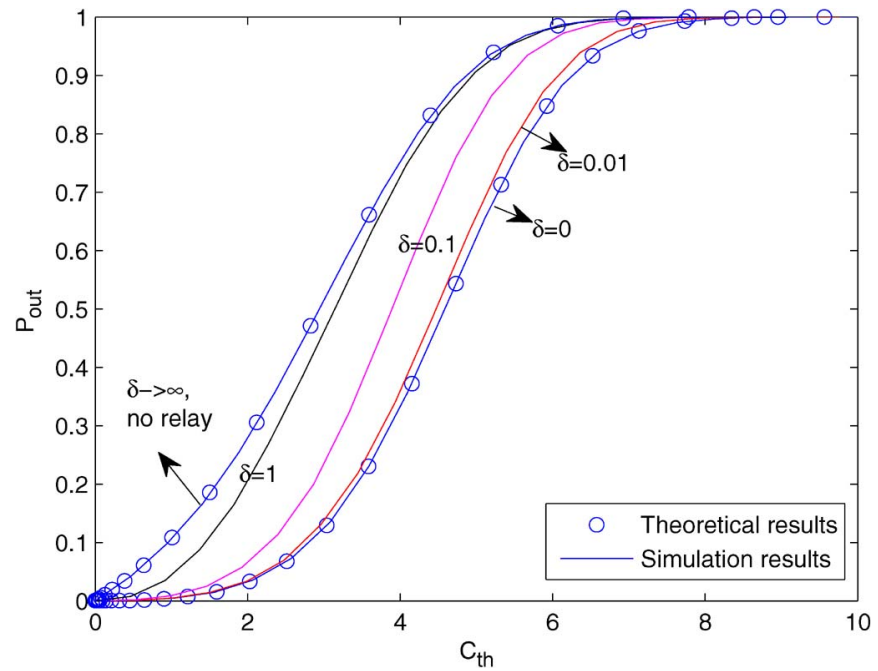

Fig. 2. Outage probability of an MS at the cell boundary when relay is available ( $\delta$ indicates the RS-MS link quality; $N=3$ ).

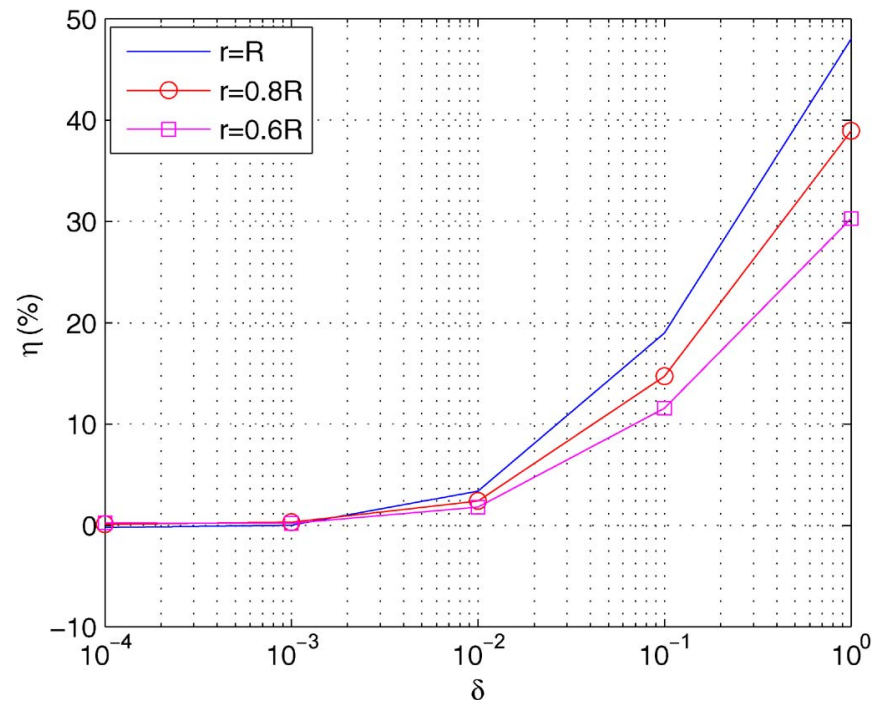

Fig. 3. Impact of $\delta$ on the outage capacity $(N=3, \epsilon=0.1)$.

shows the outage probability of an MS at $(R, 0)$ (the cell boundary) with different $\delta$. It is noted that the case of $\delta \rightarrow \infty$ is equivalent to that no relay is available, and the case of $\delta=0$ represents that the RS-MS channel is perfect. Theoretical and simulation results are both illustrated for these two extreme cases and match very well. The results indicate that the use of cognitive relay significantly improves the system performance when the RS-MS link is perfect. Simulation results for $\delta=1,0.1,0.01$ are also shown to compare with the two extreme cases. It is shown that the performance improvement is degraded with the increase in $\delta$.

To quantify the impact of $\delta$ on the system performance, Figs. 3 and 4 illustrate the degrading factor of the outage capacity for the MS at different locations $(r=R, 0.8 R, 0.6 R, \theta=0)$ when $N=3$ and $N=7$, respectively, where the degrading factor is quantified as

$$
\eta(\delta)=\frac{\left.C_{\epsilon}\right|_{\delta=0}-\left.C_{\epsilon}\right|_{\delta}}{\left.C_{\epsilon}\right|_{\delta=0}} \times 100 \% .
$$




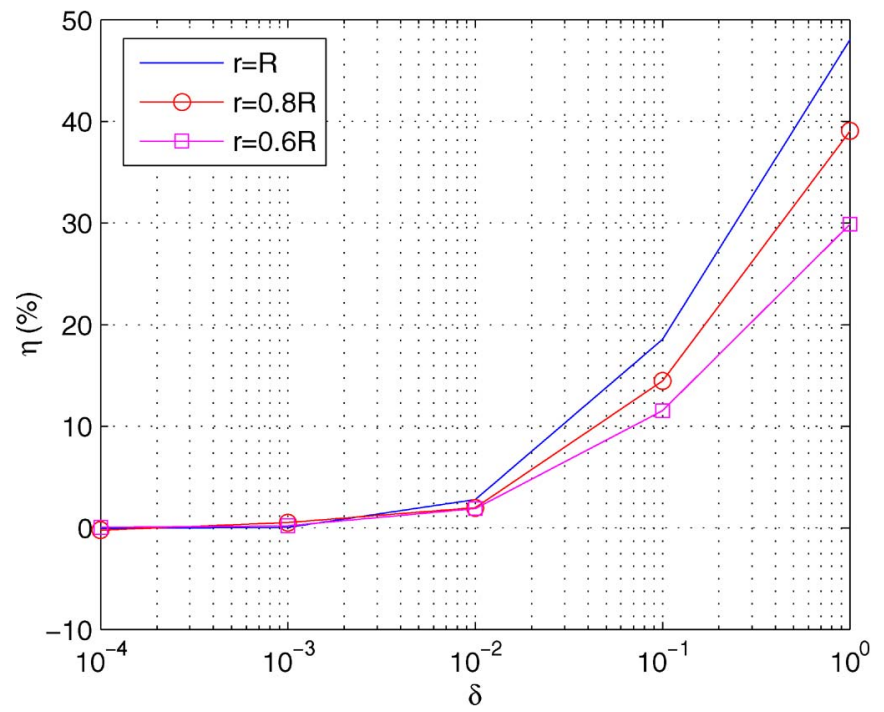

Fig. 4. Impact of $\delta$ on the outage capacity $(N=7, \epsilon=0.1)$.

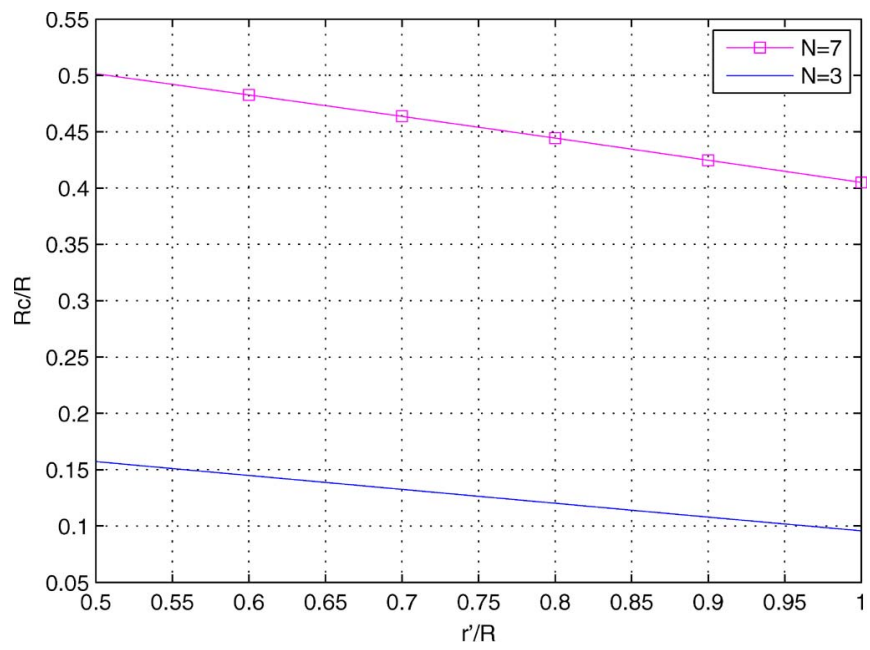

Fig. 5. Coverage radius of the RS at different locations.

The target outage probability is set to $\epsilon=0.1$. It is seen that when $\delta \leq 0.01$, the decrease in outage capacity is less than $5 \%$ for any $r$ and $N$. Therefore, we select $\delta_{0}=0.01$ in (43) so that the effect of $\delta$ is negligible.

Assume that the tolerable ISR of the neighboring MS is $\lambda=$ $-20 \mathrm{~dB}$, and the probability in (43) is $\varepsilon=0.1$. The coverage radius of the RS at different locations $\left.\left(r^{\prime}, \theta^{\prime}\right)\right)$ can be calculated following (47). Since the variation of $R_{c}$ for different $\theta^{\prime}$ is small, we simply approximate that the RS with the same $r^{\prime}$ has the same coverage radius, which is set to

$$
\tilde{R}_{c}\left(r^{\prime}\right)=\min _{\theta^{\prime}} R_{c}\left(r^{\prime}, \theta^{\prime}\right)
$$

Fig. 5 illustrates the numerical results of the coverage radius for different $r^{\prime}$ and cluster size $N$. It is seen that the coverage radius is increased with the decrease in $r^{\prime}$ and the increase in $N$. Appropriate RS locations are selected according to Fig. 5 to cover the interference-limited area. For example, assume that the interference-limited distance threshold $r_{0}=0.8 R$, i.e., all MSs with $r>0.8 R$ require the assistance of the RS. If the

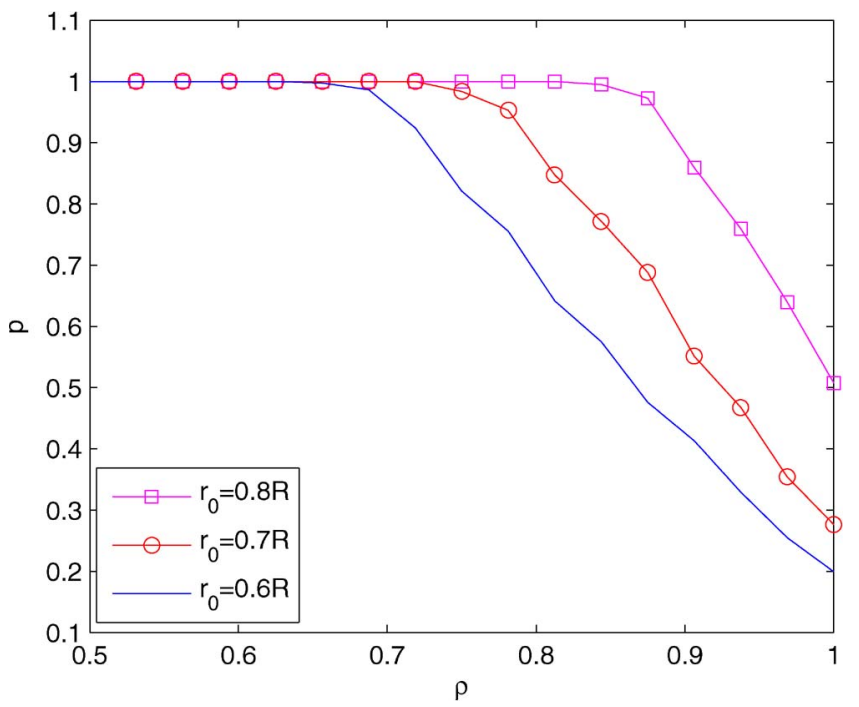

Fig. 6. Cognitive-relay channel availability probability $p$ versus loading factor $\rho$.

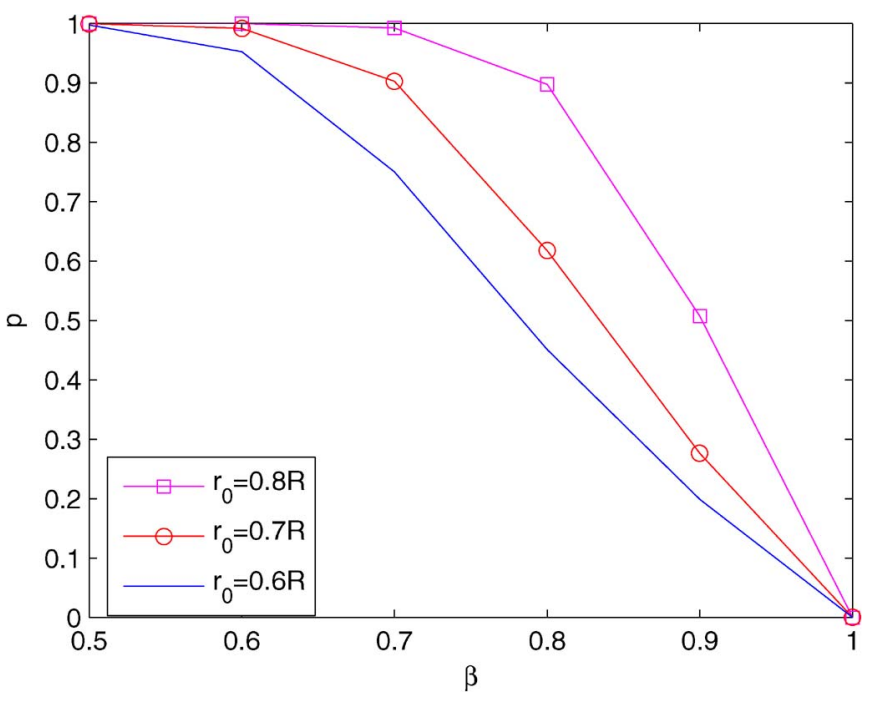

Fig. 7. Cognitive-relay channel availability probability $p$ versus activity factor $\beta$.

cluster size $N=3$, to maximize the coverage area and make sure that the cell boundary is covered, we deploy the RS at $r^{\prime}=0.9 R$. The corresponding coverage radius is $R_{c} \approx 0.1 R$. To cover all the interference-limited area, the required RS number is about $\left\lceil 2 \pi r^{\prime} / 2 R_{c}\right\rceil=28$. If $N=7$, we deploy the $\mathrm{RS}$ at $r^{\prime}=0.8 R$ to make sure that the RS is in the interferencelimited region. The corresponding coverage radius is about $R_{c}=0.45 R$. The required number of RSs is about 6 .

Furthermore, we illustrate the availability of the cognitiverelay channel in cellular systems and its effect on the outage capacity. Assume that the number of channels that are allocated to each cell is 32. Fig. 6 shows the availability probability $p$ as a function of the loading factor $\rho$ with the activity factor $\beta=0.9$, and Fig. 7 shows $p$ as a function of $\beta$ with $\rho=1$. Different threshold distances for interference-limited operation $\left(r_{0}=\right.$ $0.6 R, 0.7 R, 0.8 R)$ are considered. It is seen that $p$ is decreased with the increase in $\rho$, the increase in $\beta$, or the decrease in $r_{0}$ because the increase in $\rho$ and $\beta$ reduces the opportunity 


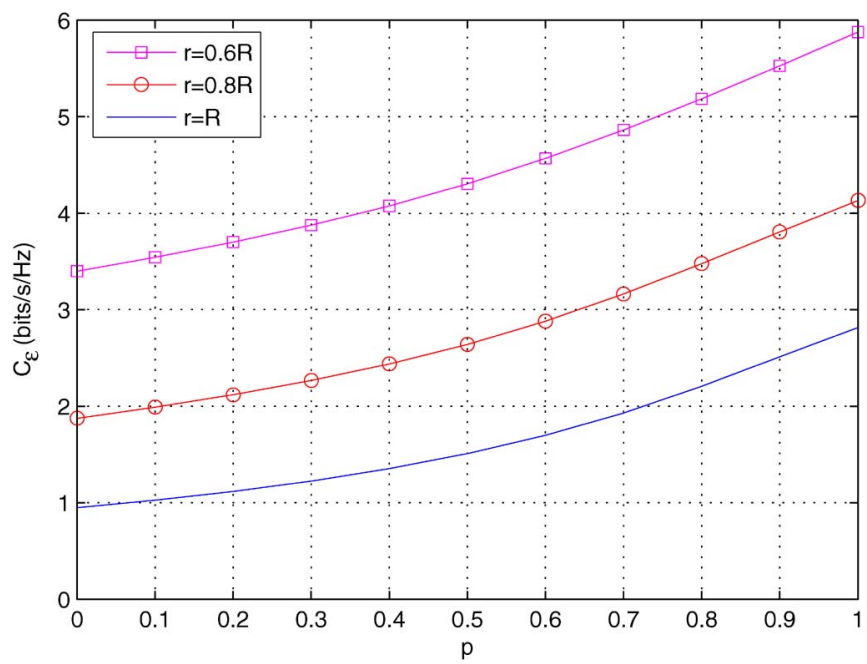

Fig. 8. Outage capacity of the MS with cognitive relay as a function of $p(N=3, \epsilon=0.1)$.

to have vacant channels, and the decrease in $r_{0}$ increases the number of interference-limited MSs, which compete for the vacant channels.

Fig. 8 shows the outage capacity of the MS at different locations in a cognitive-relay-based cellular network as a function of the channel availability probability $p$. The cluster size is $N=3$, and the outage probability is $\epsilon=0.1$. As described above, we assume that the considered MS is in the coverage area of an RS so that the RS-MS link can be regarded as perfect, i.e., $\delta=0$. From Fig. 8 , we see that if the relay channel is always available, i.e., $p=1$, the cognitive relay improves the capacity of the MS at the cell boundary by about three times compared with the system without relay, i.e., $p=0$. The improvement is decreased with the decrease in $p$. However, even when $p$ is decreased to 0.7 , the improvement brought by the cognitive-relay-based ICI cancellation still doubles the capacity of the MS at the cell boundary.

Finally, we investigate the average capacity performance of the proposed cellular system. Fig. 9 shows the average capacity of the MS at different locations and with different $\delta$ in a cognitive-relay-based cellular network as a function of the channel-availability probability $p$. It is seen that the average capacity noticeably increases with the increase in $p$. The results also confirm that the impact of $\delta$ on the system performance is very small when $\delta \leq 0.01$.

Considering Figs. 6-9, we conclude that the cognitive relay provides great opportunities to improve the capacity of cellular systems by mitigating the strong ICI near the cell boundary, particularly for systems with relatively low traffic loads.

\section{CONCLUSION}

This paper has proposed an ICI-cancellation method with the assistance of cognitive AF relay. Several RSs have been deployed around the cell boundary to opportunistically access the spectrum hole and relay signals to MSs. Optimum combining has been used at the MS to cancel the ICI. A theoretical performance analysis of the proposed system has been given in terms of the outage capacity and the average capacity. The

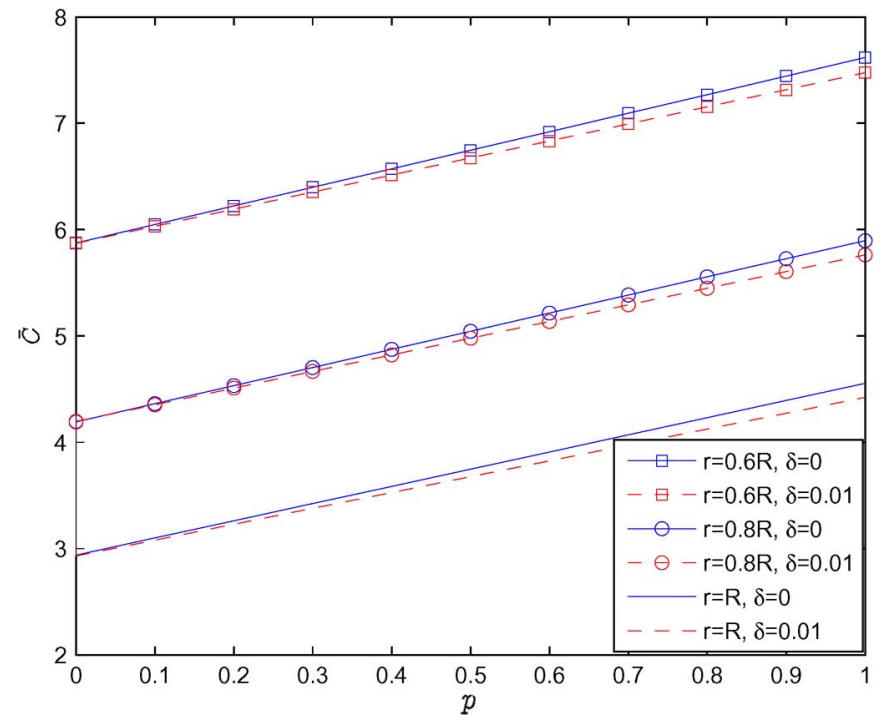

Fig. 9. Average capacity of the MS with cognitive relay as a function of $p(N=3)$.

location and the coverage area of the RS have been determined based on the performance requirements. Simulation results have been presented to validate the theoretical analysis and to show the capacity improvement that has been obtained through the ICI cancellation method using cognitive relay.

\section{REFERENCES}

[1] I. Katzela and M. Naghshineh, "Channel assignment schemes for cellular mobile telecommunication systems: A comprehensive survey," IEEE Personal Commun. Mag., vol. 3, no. 3, pp. 10-31, Jun. 1996.

[2] W. C. Y. Lee, "Overview of cellular CDMA," IEEE Trans. Veh. Technol., vol. 40, no. 2, pp. 291-302, May 1991.

[3] M. Einhaus, O. Klein, and M. Lott, "Interference averaging and avoidance in the downlink of an OFDMA system," in Proc. IEEE 16th PIMRC, Sep. 11-14, 2005, vol. 2, pp. 905-910.

[4] A. Shah and A. M. Haimovich, "Performance analysis of optimum combining in wireless communications with Rayleigh fading and cochannel interference," IEEE Trans. Commun., vol. 46, no. 4, pp. 473-479, Apr. 1998.

[5] K.-K. Wong, R. S.-K. Cheng, K. B. Letaief, and R. D. Murch, "Adaptive antennas at the mobile and base stations in an OFDM/TDMA system," IEEE Trans. Commun., vol. 49, no. 1, pp. 195-206, Jan. 2001.

[6] M. Kang, L. Yang, and M. S. Alouini, "Outage probability of MIMO optimum combining in presence of unbalanced co-channel interferers and noise," IEEE Trans. Wireless Commun., vol. 5, no. 7, pp. 1661-1668, Jul. 2006.

[7] H. Kang, J. S. Kwak, T. G. Pratt, and G. L. Stüber, "Analytical framework for optimal combining with arbitrary-power interferers and thermal noise," IEEE Trans. Veh. Technol., vol. 57, no. 3, pp. 1564-1575, May 2008.

[8] R. Pabst, B. H. Walke, D. C. Schultz, P. Herhold, H. Yanikomeroglu, S. Mukherjee, H. Viswanathan, M. Lott, W. Zirwas, M. Dohler, H. Aghvami, D. D. Falconer, and G. P. Fettweis, "Relay-based deployment concepts for wireless and mobile broadband radio," IEEE Commun. Mag., vol. 42, no. 9, pp. 80-89, Sep. 2004.

[9] H. Wu, C. Qiao, S. De, and O. Tonguz, "Integrated cellular and ad hoc relaying systems: iCAR," IEEE J. Sel. Areas Commun., vol. 19, no. 10, pp. 2105-2115, Oct. 2001.

[10] J. Cho and Z. J. Haas, "On the throughput enhancement of the downstream channel in cellular radio networks through multihop relaying," IEEE $J$. Sel. Areas Commun., vol. 22, no. 7, pp. 1206-1219, Sep. 2004.

[11] A. Sendonaris, E. Erkip, and B. Aazhang, "User cooperation diversity. Part I. System description," IEEE Trans. Commun., vol. 51, no. 11, pp. 1927-1938, Nov. 2003.

[12] A. Sendonaris, E. Erkip, and B. Aazhang, "User cooperation diversity. Part II. Implementation aspects and performance analysis," IEEE Trans. Commun., vol. 51, no. 11, pp. 1939-1948, Nov. 2003. 
[13] Q. Yang and K. S. Kwak, "Outage probability of cooperative relay in Rayleigh fading with unequal-power Rayleigh interferers," IEICE Trans. Commun., vol. E91-B, no. 10, pp. 3360-3363, Oct. 2008.

[14] K. Park, H. S. Ryu, C. G. Kang, D. Chang, S. Song, J. Ahn, and J. Ihm, "The performance of relay-enhanced cellular OFDMA-TDD network for mobile broadband wireless services," EURASIP J. Wirel. Commun. Netw., Feb. 2009, Article ID 261815.

[15] F. S. P. T. Force, Report of the Spectrum Efficiency Working Group, Nov. 2002. [Online]. Available: http://www.fcc/gov/sptf/reports.html

[16] J. Mitola and G. Q. Macguire, "Cognitive radio: Making software radios more personal," IEEE Pers. Commun., vol. 6, no. 4, pp. 13-18, Aug. 1999.

[17] K. B. Letaief and W. Zhang, "Cooperative communications for cognitive radio networks," Proc. IEEE, vol. 97, no. 5, pp. 878-893, May 2009.

[18] N. Devroye, P. Mitran, and V. Tarokh, "Achievable rates in cognitive radio channels," IEEE Trans. Inf. Theory, vol. 52, no. 5, pp. 1813-1827, May 2006.

[19] K. Lee and A. Yener, "Spectrum-sensing opportunistic wireless relay networks: Outage and diversity performance," in Proc. 40th ACSSC, Oct. 29-Nov. 1, 2006, pp. 206-210.

[20] O. Simeone, Y. Bar-Ness, and U. Spagnolini, "Stable throughput of cognitive radios with and without relaying capability," IEEE Trans. Commun., vol. 55 , no. 12 , pp. $2351-2360$, Dec. 2007.

[21] S. Kim, W. Choi, Y. Choi, J. Lee, Y. Han, and I. Lee, "Downlink performance analysis of cognitive radio based cellular relay networks," in Proc. 3rd CrownCom, May 15-17, 2008, pp. 1-6.

[22] S. Haykin, D. Thomson, and J. Reed, "Spectrum sensing for cognitive radio," Proc. IEEE, vol. 97, no. 5, pp. 849-877, May 2009.

[23] T. Yucek and H. Arslan, "A survey of spectrum sensing algorithms for cognitive radio applications," Commun. Surveys Tuts., vol. 11, no. 1, pp. 116-130, 1st Quarter, 2009.

[24] E. Larsson and M. Skoglund, "Cognitive radio in a frequency-planned environment: Some basic limits," IEEE Trans. Wireless Commun., vol. 7, no. 12, pp. 4800-4806, Dec. 2008.

[25] C. Patel and G. Stüber, "Channel estimation for amplify and forward relay based cooperation diversity systems," IEEE Trans. Wireless Commun., vol. 6, no. 6, pp. 2348-2356, Jun. 2007.

[26] M. O. Hasna and M. S. Alouini, "A performance study of dual-hop transmissions with fixed gain relays," IEEE Trans. Wireless Commun., vol. 3, no. 6, pp. 1963-1968, Nov. 2004.

[27] A. S. Avestimehr and D. N. C. Tse, "Outage capacity of the fading relay channel in the low-SNR regime," IEEE Trans. Inf. Theory, vol. 53, no. 4, pp. 1401-1415, Apr. 2007.

[28] H. Shin and M. Z. Win, "MIMO diversity in the presence of double scattering," IEEE Trans. Inf. Theory, vol. 54, no. 7, pp. 2976-2996, Jul. 2008.

[29] T. S. Rappaport, Wireless Communications: Principle and Practice, 2nd ed. Englewood Cliffs, NJ: Prentice-Hall, 2002.

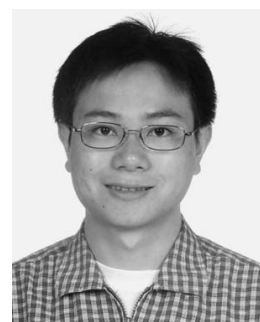

Hongbing Cheng (S'03-M'07) received the B.S. and $\mathrm{Ph} . \mathrm{D}$. degrees in electronic engineering from Peking University, Beijing, China, in 2002 and 2007, respectively.

$\mathrm{He}$ is currently a Research Assistant Professor with the Department of Electrical and Computer Engineering, Stevens Institute of Technology, Hoboken, NJ. His research interests include wireless communication theory, resource allocation for cellular and ad hoc networks, broadband code-division multiple access, cooperative relays, and cognitive radio.

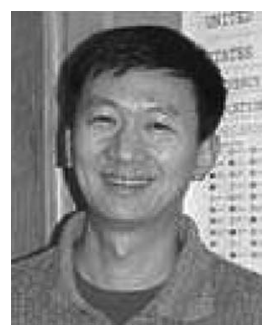

Yu-Dong Yao (S'88-M'88-SM'94) received the B.Eng. and M.Eng. degrees in electrical engineering from Nanjing University of Posts and Telecommunications, Nanjing, China, in 1982 and 1985, respectively, and the Ph.D. degree in electrical engineering from Southeast University, Nanjing, in 1988.

From 1989 to 1990, he was a Research Associate with Carleton University, Ottawa, ON, Canada, working on mobile radio communications. From 1990 to 1994, he was with Spar Aerospace Ltd., Montreal, QC, Canada, where he was involved in research on satellite communications. From 1994 to 2000, he was with Qualcomm Inc., San Diego, CA, where he participated in the research and development of wireless code-division multiple-access (CDMA) systems. Since 2000, he has been with Stevens Institute of Technology, Hoboken, NJ, where he is currently a Professor and the Chair of the Department of Electrical and Computer Engineering. He is also the Director of Stevens' Wireless Information Systems Engineering Laboratory. He is the holder of one Chinese patent and 13 U.S. patents. His research interests include wireless communications and networks, spread spectrum and CDMA, antenna arrays and beamforming, cognitive and software-defined radio, and digital signal processing for wireless systems.

Dr. Yao was an Associate Editor of the IEEE Communications Letters and the IEEE TRansactions on Vehicular Technology. He was also an Editor for the IEEE TRANSACTIONS ON WIRELESS COMMUNICATIONS. 